\title{
THOMAS BEIKLER
}

\section{Parodontitis - Einblicke in eine unterschätzte Biofilmerkrankung}

\section{Orale Biofilmerkrankungen}

Biofilme sind hochkomplexe, in der Regel oberflächenassoziierte, polymikrobielle Gemeinschaften, die strukturell in einer polymeren extrazellulären Matrix eingebettet sind. ${ }^{1}$ Es wird geschätzt, dass etwa 80 Prozent der gesamten mikrobiellen Biomasse der Erde in Biofilmen organisiert sind. Daher ist es auch nicht verwunderlich, dass etwa 75 Prozent aller Infektionskrankheiten des Menschen durch Biofilme ausgelöst und/ oder unterhalten werden. ${ }^{2}$ Innerhalb der menschlichen Mundhöhle lassen sich Biofilme an allen weich- (Schleimhäute, Zunge und Tonsillen) wie hartgewebigen (Zahn- und Zahnersatzmaterialien) Oberflächen darstellen und werden hier gemeinhin als dentale Plaque bezeichnet. ${ }^{3}$ Nahezu alle zahnmedizinisch relevanten Erkrankungen, wie beispielsweise Parodontitis, Karies, apikale Parodontitis, Peri-implantitis und die Candidiasis, sind eng mit oralen Biofilmen assoziiert. ${ }^{4}$

Die beiden häufigsten oralen Biofilmerkrankungen Karies und Parodontitis sind so alt wie die Menschheit selbst. So konnte Parodontitis-assoziierter Alverolarknochenverlust bereits bei einem drei Millionen Jahre alten Hominiden nachgewiesen werden. ${ }^{5}$ Eine der frühesten schriftlichen Belege parodontaler Erkrankungen datiert zurück in das Jahr 400 vor Christus. ${ }^{6}$ Der älteste Nachweis kariöser Läsionen stammt aus dem Mittel-Paleolithikum (70.000 bis 35.000 vor Christus). Verglichen mit heute erscheint die Kariesprävalenz zur damaligen Zeit jedoch drastisch niedriger gewesen zu sein. ${ }^{7}$ Erst mit der erhöhten Verfügbarkeit fermentierbarer Kohlenhydrate (etwa 10.000 vor Christus) stieg die Prävalenz der Karies stark an. ${ }^{8}$

In den letzten drei Jahrzehnten hat die zahnmedizinische Prävention in vielen Regionen der Welt zu einem starken Rückgang der Kariesprävalenz bei Kindern und jungen Erwachsenen geführt. ${ }^{9}$ Eine Ausnahme von diesem Trend bilden Schwellenländer wie

\footnotetext{
1 Vgl. Hojo et al. (2009).

2 Vgl. Davies (2003).

3 Vgl. Grossner-Schreiber et al. (2009), Palmer (2009), Rocha et al. (2008), Sachdeo, Haffajee und Socransky (2008) sowie Schaudinn et al. (2009).

4 Vgl. Domejean-Orliaguet, Gansky und Featherstone (2006), Flemmig (1999), Gutmann et al. (2009), Levin et al. (2009), Mariotti (1999), Meng (1999a), Meng (1999b), Novak (1999), Ramage et al. (2009), Tonetti und Mombelli (1999) sowie Zitzmann und Berglundh (2008).

5 Vgl. Langsjoen (1998).

6 Vgl. Langsjoen (1998).

7 Vgl. Forshaw (2009) sowie Leek (1966).

8 Vgl. Larsen (1995) sowie Temple und Larsen (2007).

9 Vgl. Bratthall (2005) sowie WHO (2009b).
} 
zum Beispiel Indien und China, in denen auf Grund der angestiegenen Verfügbarkeit fermentierbarer Kohlenhydrate die Kariesprävalenz stark angestiegen ist. ${ }^{10}$ Bei nahezu 100 Prozent aller Erwachsenen in Industrienationen hingegen lassen sich derzeit kariesbedingte Restaurationen nachweisen. ${ }^{11}$ Für die Zukunft ist zu erwarten, dass die Abnahme der Kariesprävalenz in der jüngeren Alterskohorte in Industrienationen zu weniger Extraktionen in der mittleren und älteren Altersgruppe führt. ${ }^{12}$ Da mit zunehmendem Alter Risikofaktoren für Karies ansteigen, ${ }^{13}$ ist zu vermuten, dass sich die Kariesprävalenz in der älteren Bevölkerungsgruppe zukünftig erhöhen wird. ${ }^{14}$

Im Vergleich zur Karies zeigt die globale Prävalenzentwicklung der Parodontitis einen deutlich abweichenden Trend. In weiten Teilen Afrikas, Asiens, Europas, Nordund Südamerikas zeigt sich die Prävalenz der Parodontitis nahezu unverändert. Für Deutschland wurde sogar eine signifikante Zunahme parodontaler Erkrankungen in den letzten Jahrzehnten gezeigt. Durchschnittlich sind etwa 5 bis 20 Prozent der Erwachsenen und etwa 2 Prozent der Jugendlichen einer Population von einer schweren Parodontitis betroffen. ${ }^{15}$ Bei mehr als 50 Prozent einer Population lässt sich parodontaler Attachmentverlust nachweisen.

Diese Daten deuten darauf hin, dass die derzeit durchgeführten zahnmedizinischen Prophylaxestrategien aus parodontologischer Sicht als unzureichend bezeichnet werden müssen. Dies kann unter anderem damit erklärt werden, dass die Pathogenese der Parodontitis - im Gegensatz zur Karies - bisher nicht ausreichend verstanden wurde und somit spezifische Ansätze zur Prävention parodontaler Erkrankungen fehlen. Daher konzentriert sich die Arbeit im Folgenden auf die Darstellung der bisherigen Informationen zur Pathogenese der Parodontitis.

\section{Mikrobiologische Aspekte der Parodontitis}

Trotz enormer Forschungsfortschritte im Bereich der oralen Mikrobiologie ist die Rolle des intraoralen Biofilms in der Pathogenese der Parodontitis bisher nur unzureichend verstanden. Dies liegt daran, dass die Parodontitis nicht von einer bakteriellen Spezies initiiert und unterhalten wird, sondern in der Tat das Ergebnis einer polymikrobiellen Kolonisation intraoraler Oberflächen darstellt. Darüber hinaus sind die intraoralen Kolonisationsmuster von Patienten mit Parodontitis individuell höchst unterschiedlich, und zum Teil lassen sich als parodontopathogen klassifizierte Erreger auch bei parodontal gesunden Patienten nachweisen. Den klassischen Koch'schen Postulaten folgend erlauben diese Umstände nicht, einen Mikroorganismus eindeutig als Auslöser einer Parodontitis zu bezeichnen. Aus diesem Grund wurde das Koch'sche Postulat bereits 1992 von Socransky und Haffajee modifiziert. ${ }^{16}$ Als parodontopathogen wurde demnach derjenige Erreger bezeichnet, ${ }^{17}$ der

\footnotetext{
$\overline{10}$ Vgl. Bratthall (2005) sowie Petersen et al. (2005).

${ }^{11}$ Vgl. Dye et al. (2007).

12 Vgl. Baelum et al. (2007).

${ }^{13}$ Vgl. Hintao et al. (2007), Imazato et al. (2006) sowie Le Pera, Mahevich und Silverstein (2005).

${ }^{14}$ Vgl. Hugoson et al. (2005a) sowie Hugoson et al. (2005b).

${ }^{15}$ Vgl. Albandar, Brown und Loe (1997), Petersen et al. (2005) sowie WHO (2009a).

${ }^{16}$ Vgl. Socransky und Haffajee (1992).

${ }^{17}$ Vgl. Feng und Weinberg (2006).
} 
- $\quad$ sich in hoher Anzahl in oder um parodontale Läsionen nachweisen lässt;

- sich im Vergleich zu Parodontitispatienten in niedriger Anzahl bei parodontal gesunden Probanden oder Personen mit anderen Formen parodontaler Erkrankungen nachweisen lässt;

- $\quad$ eine starke humorale Immunreaktion bei parodontal Erkrankten induziert;

- In-vitro-Virulenzfaktoren exprimiert, die mit der klinischen Histopathologie vereinbar sind,

- und der im Tiermodell vergleichbare pathogene Fähigkeiten besitzt.

Der Einsatz moderner mikrobiologischer Analyseverfahren hat allerdings gezeigt, dass innerhalb derselben Spezies eine ausgeprägte genetische Heterogenität existiert. Obwohl Angehörige derselben Spezies über eine Reihe gemeinsamer und damit identischer Housekeeping-Gene verfügen, zeigen sie jedoch häufig quantitative und qualitative Unterschiede im Genom. In diesem Zusammenhang haben Genomanalysen innerhalb von Stämmen derselben Spezies ergeben, dass nur etwa zwei Drittel des gesamten Genoms identisch ist, ${ }^{18}$ der Rest der variablen Gene codiert in der Regel Proteine, die die Anpassungsfähigkeit und/oder Virulenz der Organismen erhöhen. ${ }^{19}$ Dies mag eine Erklärung sein für die Tatsache, dass die Erregerprofile bei Erkrankten unterschiedlich sind ${ }^{20}$ und manche Individuen auch als parodontopathogen bezeichnete Bakterien intraoral beherbergen können, ohne dass klinische Zeichen einer Parodontitis vorliegen. Daher müssen bakterielle Spezies als Populationen genetisch unterschiedlicher Klone betrachtet werden, was eine Verallgemeinerung hinsichtlich des speziesspezifischen pathogenen Potentials unmöglich macht. ${ }^{21}$ Aus diesem Grund wurde das von Socransky vorgeschlagene Modell im Laufe der Zeit um den Faktor Klonalität erweitert. ${ }^{22}$

Entsprechend der oben dargelegten Definition wird Aggregatibacter actinomycetemcomitans (A. actinomycetemcomitans) als eng mit der aggressiven Parodontitis assoziiert betrachtet. ${ }^{23}$ Eine bedeutende Rolle in der Ätiopathogenese der chronischen Parodontitis spielen neben A. actinomycetemcomitans Keime wie Porphyromonas gingivalis (P. gingivalis), Tannerella forsythensis (T. forsythensis), Prevotella intermedia (P. intermedia), Campylobacter rectus (C. rectus) und Treponema denticola (T. Denticola). ${ }^{24}$ In diesem Zusammenhang sei allerdings darauf hingewiesen, dass der Nachweis von parodontopathogenen Keimen wie A. actinomycetemcomitans, $P$. gingivalis, $P$. intermedia, $C$. rectus und $T$. forsythensis keine Unterscheidung zwischen aggressiver und chronischer Parodontitis erlaubt. ${ }^{25}$

\footnotetext{
${ }^{18}$ Vgl. Hotopp et al. (2006) sowie Tettelin et al. (2005).

${ }^{19}$ Vgl. Fraser, Hanage und Spratt (2005).

${ }^{20}$ Vgl. Beikler et al. (2004a) sowie Beikler et al. (2004b).

${ }^{21}$ Vgl. Beikler et al. (2003), Chen et al. (2004), Haubek, Poulsen und Kilian (2007) sowie Inaba et al. (2008).

${ }^{22}$ Vgl. Feng und Weinberg (2006) sowie Nishihara und Koseki (2004).

${ }^{23}$ Vgl. Slots und Ting (1999).

${ }^{24}$ Vgl. Colombo et al. (2009), Ezzo und Cutler (2003), Feng und Weinberg (2006), Haffajee et al. (2008), Socransky und Haffajee (1992), Socransky und Haffajee (2005), Socransky et al. (1998), Tanner et al. (2007), Teles et al. (2008) sowie Ximenez-Fyvie et al. (2000).

${ }^{25}$ Vgl. Feng und Weinberg (2006) sowie Mombelli, Casagni und Madianos (2002).
} 


\section{Parodontopathogene Erregercluster}

Der Übergang zur Krankheit wird begleitet von einer Transition des subgingivalen Ökosystems, das sich von einer (fakultativ) aeroben, fermentierenden, gram-positiven hin zu einer anaeroben, proteolytischen, gram-negativen Mikroflora entwickelt. ${ }^{26}$ Die wegweisenden Arbeiten von Socransky et al. haben gezeigt, dass parodontopathogene Erreger häufig nicht isoliert, sondern zusammen in spezifischen Gruppen oder Konsortien auftreten. ${ }^{27}$ Bisher konnten in subgingivalen Bereichen fünf verschiedene mikrobiologische Komplexe identifiziert werden, ${ }^{28}$ die eng mit der parodontalen Situation assoziiert erscheinen. Ein gelber Komplex (bestehend aus Streptococci spp., wie beispielsweise $S$. sanguis und Streptococcus oralis) bildet zusammen mit einem violetten Komplex (bestehend aus Actinomyces odontolyticus und Veillonella parvula) die Gruppe der Frühbesiedler, die adhäsionsrelevante Rezeptoren exprimieren und so ein rasches Anheften an Oberflächen ermöglichen. Ein grüner Komplex (bestehend aus Capnocytophaga spp., Campylobacter concisus, Eikenella corrodens und A. actinomycetemcomitans Serotyp a) kann ebenfalls - vor allem im supragingivalen Biofilm ${ }^{29}$ - bei Parodontitispatienten nachgewiesen werden, scheint jedoch weniger stark mit anderen parodontopathogenen Erregern assoziiert zu sein. ${ }^{30}$ Das Vorkommen eines roten Komplexes (bestehend aus $P$. gingivalis, $T$. forsythensis und $T$. denticola) und eines orangefarbigen Komplexes (unter anderen Prevotella nigrescens, Prevotella intermedia, Peptostreptococcus micros, verschiedene Fusobacterium spp. und eng assoziiert Campylobacter spp., Streptococcus constellatus, Eubacterium nodatum) ist oft mit erhöhter Taschensondiertiefe (TST) und Blutung auf Sondierung (BAS) verbunden. ${ }^{31}$ Interessanterweise steigt die Detektionsfrequenz von Bakterien, die sich dem roten und orangefarbigen Komplex zuordnen lassen, mit steigender Taschensondiertiefe signifikant $\mathrm{an}^{32}$. Die Spezies des orangefarbigen Komplexes werden auch als „Brückenspezies“ bezeichnet, da sie sowohl Bindungsrezeptoren für die Frühbesiedler als auch für die Spätbesiedler des roten Komplexes ausbilden. ${ }^{33}$ Auch der violette Komplex, der eng mit dem orangefarbigen Komplex verbunden ist, wird gehäuft bei entzündeten Parodontien beobachtet. ${ }^{34}$

\section{Parodontopathogene Erreger und Progression der Parodontitis}

Verschiedene longitudinale Langzeitstudien haben gezeigt, dass der intraorale Nachweis, wie erhöhte Titer von $P$. gingivalis, A. actinomycetemcomitans, $T$. forsythensis und $P$. Intermedia, als Indikator zukünftiger Attachment- und Alveolarknochenverlusten bei Individuen mit chronischer oder aggressiver Parodontitis dienen kann. ${ }^{35}$ Eine erhöhte Detektionsfrequenz von P. gingivalis, A. actinomycetemcomitans und T. forsythen-

\footnotetext{
${ }^{26}$ Vgl. Feng und Weinberg (2006).

${ }^{27}$ Vgl. Socransky et al. (1998).

${ }^{28}$ Vgl. Socransky et al. (1998).

${ }^{29}$ Vgl. Ximenez-Fyvie et al. (2000).

${ }^{30}$ Vgl. Holt und Ebersole (2005).

${ }^{31}$ Vgl. Holt und Ebersole (2005) sowie Socransky et al. (1998).

32 Vgl. Riviere et al. (1996) sowie Socransky und Haffajee (2005).

${ }^{33}$ Vgl. Kolenbrander et al. (2002).

${ }^{34}$ Vgl. Feng und Weinberg (2006).

${ }^{35}$ Vgl. Haffajee et al. (1991), Machtei et al. (1997), Machtei et al. (1999), Timmerman et al. (2000) sowie Tran et al. (2001).
} 
sis in Stellen mit progredienten Attachmentverlusten konnte bei Patienten mit mittelschwerer bis schwerer chronischer Parodontitis nachgewiesen werden. ${ }^{36}$ In einer interessanten longitudinalen Langzeitstudie von Tanner et al. konnte P. gingivalis und T. forsythensis in Individuen mit generalisierter leichter chronischer Parodontitis und progredienten flachen Taschen signifikant häufiger nachgewiesen werden als in Patienten mit stabilen parodontalen Verhältnissen. ${ }^{37}$ Der kontinuierliche subgingivale Nachweis von $T$. forsythensis über einen Zeitraum von zwei Jahren in Patienten mit leichter generalisierter chronischer Parodontitis ist mit einem höheren Risiko (OR = 5,3) für einen Alveolarknochenverlust verbunden. ${ }^{38}$ Beide Ergebnisse deuten drauf hin, dass $P$. gingivalis und $T$. forsythensis möglicherweise eine besondere Rolle in der Frühphase einer Parodontitis spielen. Für A. actinomycetemcomitans existieren einige interessante Studien, die deutlich zeigen, dass spezifische Klone dieses Bakteriums stärker mit aggressiven Parodontitiden assoziiert zu sein scheinen als andere. Hierunter werden die stark leukotoxinproduzierenden JP2 sowie cdt (cytolethal distending toxin) tragende Klone gezählt. ${ }^{39}$ Neben A. actinomycetemomitans scheinen bei lokalisierter Parodontitis auch P. gingivalis, T. forsythensis, Treponema denticola und Camphylocabter gracilis, Eubacterium nodatum und Prevotella intermedia bei der Progression lokalisierter aggressiver Parodontitiden eine Rolle zu spielen. ${ }^{40}$

Die klinische Bedeutung mikrobiologischer Befunde im Hinblick auf die Progression parodontaler Attachmentverluste kann allerdings nicht abschließend beantwortet werden. Dies liegt vor allem an den bereits oben gemachten Aussagen über die genetische Heterogenität parodontopathogener Erreger sowie das bisher unzureichende Wissen über die Ursachen der individuellen Prädisposition ${ }^{41}$ bei ansonsten systemisch gesunden Patienten.

\section{Immunologische/genetische Aspekte der Parodontitis}

Das heterogene klinische Bild einer Parodontitis und das Ausmaß der parodontalen Zerstörung werden neben der Kolonisation des kommensalen intraoralen Biofilms mit parodontopathogenen Keimen auch eng mit der individuellen Prädisposition des Wirtes verknüpft. Bisher sind eine Reihe von endogenen (Diabetes mellitus, primäre und sekundäre Immundefekte) wie exogene Risikofaktoren (Nikotinmissbrauch, bestimmte Arzneimittel) für die Parodontitis identifiziert worden. Im Fall des Diabetes mellitus konnte eindeutig gezeigt werden, dass die Wahrscheinlichkeit für das Auftreten einer Parodontitis bei Diabetikern mit einer OR von zwei bis drei deutlich erhöht ist. ${ }^{42}$ Der wahrscheinlich größte exogene Risikofaktor für eine Parodontitis ist mit einem dosisabhängigen relativen Risiko von fünf bis sechs der inhalative Nikotinmissbrauch. ${ }^{43}$

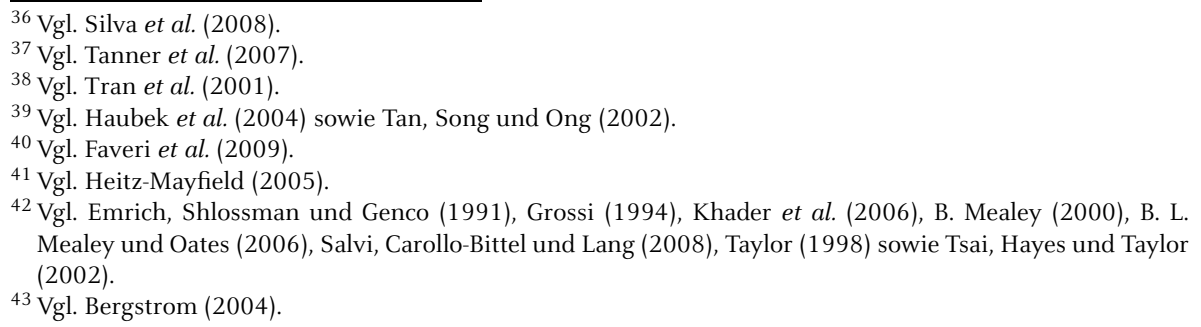


Die oben angegebenen Faktoren können aber immer noch keine ausreichende Erklärung für die hohe Prävalenz der Parodontitis in den unterschiedlichsten Populationen sein. Ergebnisse von Zwillingsuntersuchungen deuten an, dass ungefähr 50 Prozent parodontaler Attachmentverluste einer genetischen Prädisposition zugeschrieben werden können. ${ }^{44}$ Mutationen in einzelnen Genen - wie beispielsweise für das CTSG-Gen im Rahmen des Papillon-Lefevre Syndrom beschrieben - sind allerdings außerordentlich selten. ${ }^{45}$ Es wird vermutet, dass mindestens zehn bis zwanzig Gene direkt Einfluss auf das Entstehen und das Fortschreiten aggressiver wie chronischer Parodontitiden nehmen. Allerdings brachten die bisherigen Versuche, diese Gene zu identifizieren, keine richtungsweisenden Ergebnisse. ${ }^{46}$

Unstrittig ist jedoch, dass eine Dysregulation auf immunologisch-inflammatorischer Ebene eine bedeutende Rolle für das individuell unterschiedliche klinische Bild einer Parodontitis spielen muss. Diese wird durch Vorgänge im Rahmen der unspezifischen Immunabwehr, der nachfolgenden Aktivierung von Entzündungsprozessen und der spezifischen Immunabwehr charakterisiert. Gesteuert werden diese Prozesse durch ein komplexes Netzwerk pro-inflammatorischer Zytokine und Mediatoren myeloischer und nicht myeloischer Zellen, die entweder die Entzündung initiieren oder aber Toleranz gegenüber den als parodontopathogen bezeichneten Bakterien induzieren.

Die Klärung dieser Vorgänge ist derzeit ein zentraler Fokus der parodontalen Forschung weltweit. Neben unzähligen In-vitro-Studien sind allerdings bisher nur vereinzelt Arbeiten zur Expression inflammatorischer Prozesse in vivo erschienen. Alle diese Arbeiten wurden in unbehandelten Geweben von Parodontitispatienten durchgeführt und nicht mit dem Gewebe parodontal Gesunder verglichen. Die Prävalenz der akuten Entzündung erschwert die Identifikation parodontitisspezifischer pathogener Mechanismen. Aus diesem Grund wurden Biopsien bei Patienten nach nicht-chirurgischer Therapie entnommen und mit parodontal gesundem Gewebe verglichen. Mittels Microarray und RT-PCR basierter Analysen konnte im parodontal erkrankten Gewebe eine Reihe von Genen signifkant up- beziehungsweise downreguliert beobachtet werden. Mittels spezifischer Software konnten folgende Beziehungen zwischen diesen auf den ersten Blick nicht zusammenhängenden Faktoren konstruiert werden. Einer der festgestellten Zusammenhänge erscheint besonders interessant und soll hier kurz umrissen werden (siehe Abb. 1):

Das in unserer Studie ${ }^{47}$ stark erhöht exprimierte IL-12 ist bekanntermaßen ein Produkt verschiedener an Entzündungsgeschehen beteiligter Zellen (Monozyten, Makrophagen, neutrophilen Granulozyten und dendritischer Zellen) und induziert die Differentierung von T-Helfer-Zellen $\left(\mathrm{T}_{\mathrm{H}} 1\right)$. Dies wird unterstützt durch die Beobachtung einer erhöhten Expression von CD38, das klassischerweise auch bei frühen T-Zell-Vorläufern und aktivierten reifen T-Zellen nachgewiesen werden kann. ${ }^{48}$ Parallel dazu konnte eine erhöhte Expression von RCAN-1 festgestellt werden. RCAN-1 inhibiert die Dephosphorylierung des Nuclear Factors of activated T-cells (NFAT) durch Calcineu-

\footnotetext{
${ }^{44}$ Vgl. Michalowicz et al. (2000).

${ }^{45}$ Vgl. Loos, John und Laine (2005) sowie Toomes et al. (1999).

${ }^{46}$ Vgl. Loos et al. (2005) sowie Tabor, Risch und Myers (2002).

${ }^{47}$ Vgl. Beikler et al. (2008).

${ }^{48}$ Vgl. Sandoval-Montes und Santos-Argumedo (2005).
} 
rin und führt dadurch zu verminderter T-Zell-Aktivierung. ${ }^{49}$ Der Wirkmechanismus ist vergleichbar mit dem Immunsuppressivum Cyclosporin A. Das für RCAN-1 codierende Gen ist auf dem Chromosom 21 lokalisiert und zeigt sich bei Patienten mit Trisomie 21 um den Faktor 20 bis 30 überexprimiert. Trisomie-21-Patienten leiden häufig an einer schweren generalisierten aggressiven Parodontitis. Die klinischen Symptome bei Trisomie-21-Patienten sowie die Beobachtung einer verstärkten Expression bei Patienten mit chronischer Parodontitis machen dieses Protein interessant für weitere Untersuchungen mit dem Ziel, die individuell unterschiedliche Prädisposition für parodontale Erkrankungen zu klären. Neueste eigene Untersuchungen in vitro zeigen, dass die Expression von RCAN-1 durch parodontopathogene Erreger wie P. gingivalis stimuliert werden kann. Diese Daten deuten an, dass parodontopathogene Keime direkt die lokale Immunantwort negativ beeinflussen und damit einer Ausbreitung der parodontalen Infektion Vorschub leisten können.

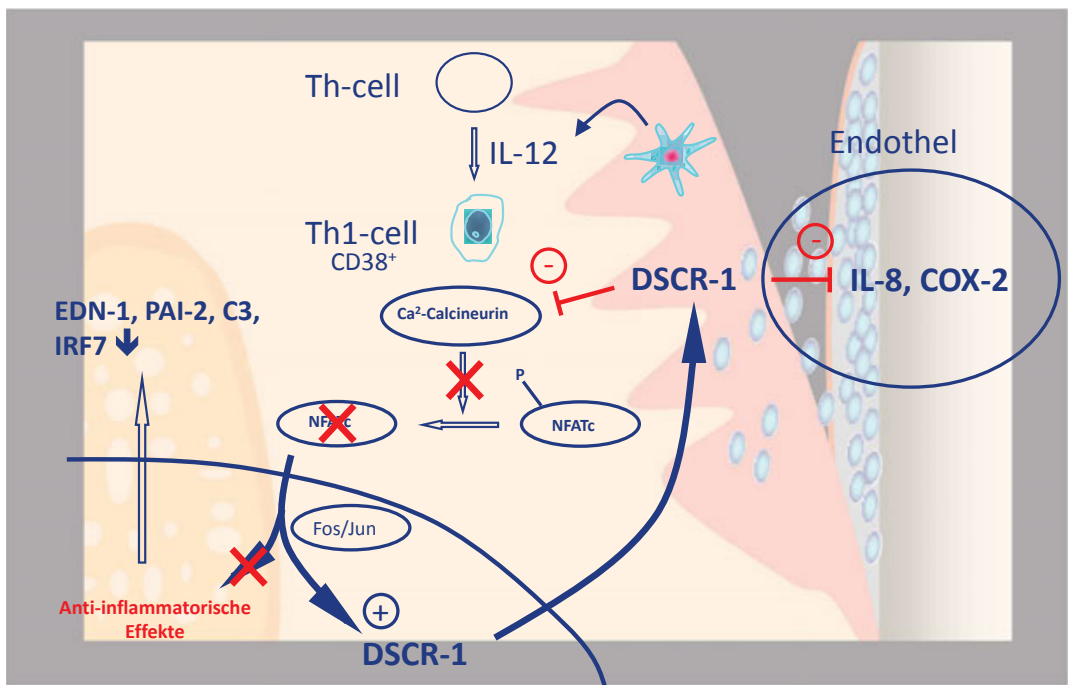

Abb. 1: Pathways der parodontalen Immun-/Entzündungsantwort

\section{Lokale Folgen oraler Biofilmerkrankungen}

Orale Biofilmerkrankungen wie Karies und Parodontitis sind weltweit die häufigste Ursache für Zahnverlust. Etwa 76 Prozent aller Extraktionen werden aus parodontalen oder kariologischen Gründen durchgeführt. ${ }^{50}$ Die Zahl der vorhandenen Zähne hat

\footnotetext{
${ }^{49}$ Vgl. Kim et al. (2006).

${ }^{50}$ Vgl. Agerholm und Sidi (1988), Aida et al. (2006), Al-Shammari et al. (2006), Angelillo, Nobile und Pavia (1996), Cahen, Frank und Turlot (1985), Chauncey, Glass und Alman (1989), Kay und Blinkhorn (1986), Klock und Haugejorden (1991), McCaul, Jenkins und Kay (2001), Murray et al. (1997), Ong, Yeo und Bhole (1996), Phipps und Stevens (1995), Reich und Hiller (1993), Richards et al. (2005), Stephens, Kogon und Jarvis (1991) sowie Trovik, Klock und Haugejorden (2000).
} 
einen weitreichenden Einfluss auf das Individuum. Neben der funktionellen Bedeutung (Nahrungsaufnahme) kommt den Zähnen auch eine große psychosoziale Bedeutung (Kommunikation) zu. ${ }^{51}$

\section{Systemische Folgen oraler Biofilmerkrankungen}

Orale Biofilmerkrankungen können durch lokales Übergreifen auf benachbarte Gewebe, hämatogene bakterielle Streuung und durch inflammatorische Mechanismen systemische Auswirkungen nach sich ziehen.

Akute Formen odontogener Infektionen können in benachbarte Gewebe übergreifen und dort zu Osteomyelitis, Sinusitis, Abszessen, Septikämie und durchaus auch zu Todesfällen führen. ${ }^{52}$ So liegt die Mortalitätsrate unbehandelter ins Mediastinum absteigender Infektionen oder Nomas bei etwa 50 bis 80 Prozent. ${ }^{53}$ Es ist daher nicht erstaunlich, dass im Mittelalter Karies und Parodontitis mit einem erhöhten Todesfallrisiko assoziiert waren. ${ }^{54}$ Kirchenbucheinträge aus dem 18. und 19. Jahrhundert belegen, dass "Zahnfieber" für etwa 30 Prozent der Todesfälle im Kindesalter verantwortlich war. ${ }^{55}$ Obwohl der medizinische Fortschritt zu einer starken Reduktion der durch dentale Ursachen bedingten Mortalitätsrate geführt hat, sind auch noch heute allein in den USA pro Jahr etwa 21.000 stationäre Aufenthalte und etwa 150 Todesfälle durch dentale Ursachen bedingt. ${ }^{56}$ Etwa 770.000 Fälle des lebensbedrohlichen Nomas werden pro Jahr weltweit registriert. ${ }^{57}$

Die in den Biofilmen der Mundhöhle befindlichen Bakterien können hämatogen potentiell in alle Regionen des Organismus verschleppt werden, wie mikrobiologische Untersuchungen von Infektionen fernab der Mundhöhle gezeigt haben. ${ }^{58}$ Eine hämatogene Streuung erscheint allerdings eher selten zu sein, wie Fallberichte zeigen, die Bakterien der Mundflora bei Endokarditis, akuter bakterieller Myokarditis, Abszessgeschehen der Leber, Lunge und des Gehirns, Thrombosen des sinus cavernosus und bei Infektionen künstlicher Gelenke nachgewiesen haben. ${ }^{59}$

Die Vermutung, dass die Parodontitis direkt Einfluss auf den Gesamtorganismus durch Modulation inflammatorischer Prozesse nimmt, wird durch Querschnittsuntersuchungen unterstützt, die erhöhte systemische Inflammationsmarker bei Patienten mit Parodontitis festgestellt haben. ${ }^{60}$ Eine Vielzahl von Untersuchungen konnte eine enge Assoziation von Parodontitis und kardiovaskulären und cerebrovaskulären Krankheitsbildern sowie dem Diabetes mellitus unabhängig von gemeinsamen Risikofaktoren wie

\footnotetext{
${ }^{51}$ Vgl. Elias und Sheiham (1999), Yoshida et al. (2001), Sheiham et al. (2001), Akifusa et al. (2005) sowie Gotfredsen und Walls (2007).

${ }^{52}$ Vgl. Bomeli, Branstetter und Ferguson (2009), Enwonwu, Falkler und Idigbe (2000), Parahitiyawa et al. (2009), Robertson und Smith (2009), Sharkawy (2007) sowie Vieira et al. (2008).

53 Vgl. Marck (2003).

${ }^{54}$ Vgl. Dewitte und Bekvalac (2009).

55 Vgl. Alt (2006).

${ }^{56}$ Vgl. Topazian et al. (2002).

${ }^{57}$ Vgl. Baratti-Mayer et al. (2003) sowie Mignogna und Fedele (2006).

${ }^{58}$ Vgl. Li et al. (2000).

${ }^{59}$ Vgl. Bartzokas et al. (1994), Mueller et al. (2009), Mylonas et al. (2007), Parahitiyawa et al. (2009), Ulivieri, Oliveri und Filosomi (2007), Wagner et al. (2006) sowie Wilson et al. (2007).

${ }^{60}$ Vgl. Chapple (2009), Craig et al. (2003), Deliargyris et al. (2004), Linden et al. (2008) sowie Slade et al. (2000).
} 
Rauchen, Alter, Schulbildung, Body-Mass-Index und Lebensführung nachweisen. ${ }^{61}$ Das relative Risiko für koronare Ereignisse ist bei Patienten mit Parodontitis um den Faktor 1,5 bis 2 erhöht; ${ }^{62}$ das adjustierte Risiko für einen Apoplex liegt bei 7,4. ${ }^{63}$ Bei Diabetikern konnte eine gleichzeitig vorliegende unbehandelte Parodontitis als Risikofaktor für eine schlechte Blutzuckerkontrolle identifiziert werden ${ }^{64}$. Der Einfluss der Parodontitis auf Frühgeburtlichkeit, Osteoporose, Neoplasien und chronisch obstruktive Lungenerkrankungen wird kontrovers diskutiert und kann daher nicht abschließend beurteilt werden. ${ }^{65}$ Die Therapie einer Parodontitis scheint umgekehrt einen Einfluss auf andere Erkrankungen zu haben. So konnte gezeigt werden, dass eine Parodontitistherapie dazu beitragen kann, die Blutzuckereinstellung zu verbessern. Ferner konnte festgestellt werden, dass in Folge einer Parodontitistherapie der Inflammationsstatus von Endothelien positiv beeinflusst wird und sich eventuell auch ein positiver Effekt auf Zustandekommen und Progression inflammatorisch induzierter Gefäßkrankheiten vermuten lässt. ${ }^{66}$ Der direkte Nachweis, dass eine Parodontitistherapie das Risiko für kardiovaskuläre Erkrankungen reduziert, steht jedoch noch aus.

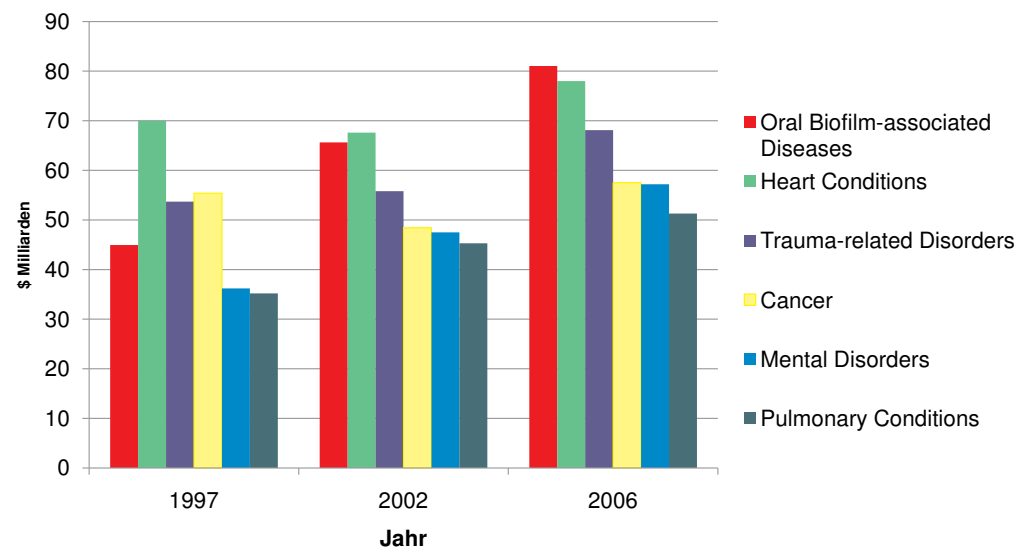

Abb. 2: Ausgaben für verschiedene Krankheitsgruppen in den USA aus den Jahren 1997, 2002 und 2006. Die Zahlen beinhalten alle Aufwendungen, einschließlich Privatleistung der Patienten, Zahlungen von privaten Krankenkassen, Medicaid und Medicare.

\section{Bedeutung und Ausblick}

Mit 81 Milliarden US-Dollar zeigt sich die Behandlung oraler Biofilmerkrankung als die kostenintensivste Erkrankung in den USA. Im Jahr 2006 rangierte das Ausgabenvolumen noch vor den Aufwendungen für kardiovaskuläre (78,0 Milliarden US-Dollar),

\footnotetext{
${ }^{61}$ Vgl. Persson und Persson (2008).

${ }^{62}$ Vgl. Beck et al. (1996) sowie Dietrich et al. (2008).

${ }^{63}$ Vgl. Collin et al. (1998) sowie Dorfer et al. (2004).

${ }^{64}$ Vgl. Collin et al. (1998), Lim et al. (2007), Nelson et al. (1990) sowie Taylor (1998).

${ }^{65}$ Vgl. Azarpazhooh und Leake (2006), Fitzpatrick und Katz (2009), Kim und Amar (2006) sowie Wimmer und Pihlstrom (2008).

${ }^{66}$ Vgl. Garcia (2009) sowie Tonetti (2009).
} 
onkologische (57,5 Milliarden US-Dollar), trauma-assoziierte (68,1 Milliarden US-Dollar), psychische (57,2 Milliarden US-Dollar) und pulmonale Erkrankungen (51,3 Milliarden US-Dollar; siehe Abb. 2). ${ }^{67}$ Obwohl Vergleichsdaten aus Deutschland fehlen, ist davon auszugehen, dass sich die Ausgaben für die zahnmedizinische Rehabilitation in einer ähnlichen Relation darstellen. In den letzten Jahrzehnten wurden substantielle Fortschritte im Verständnis der Ätiologie und Pathogenese oraler Biofilmerkrankungen gemacht, dennoch zeigen die genannten Zahlen eindrücklich, dass die bisherigen Strategien zur Prävention oraler Biofilmerkrankungen scheinbar nicht spezifisch genug sind. Um die Prävalenz oraler Biofilmerkrankungen weiter zu reduzieren, ist eine alleinige Erhöhung der Anzahl an Mundhygieneprodukten oder eine stärkere Aufklärung der Bevölkerung nicht ausreichend. Verfahren und Strategien, die weniger von der individuellen Mitarbeit des Patienten abhängig sind, scheinen erforderlich, um die Kontrolle über orale Biofilmerkrankungen zu gewinnen. Ansätze, die Transmission oralpathogener Erreger oder deren Adhäsion an oralen Oberflächen zu verhindern, sowie die Etablierung einer für oralpathogene Erreger „unattraktiven“ Mundflora könnten den Durchbruch auf diesem Gebiet ermöglichen. Erste Ergebnisse der Düsseldorfer Forschung wurden bereits patentiert und werden gegenwärtig, unterstützt durch qualifizierte Drittmittel, in klinischen Untersuchungen evaluiert.

\section{Literatur}

Agerholm, D. M. und A. D. Sidi (1988). „Reasons given for extraction of permanent teeth by general dental practitioners in England and Wales", British Dental Journal 164(11), 345-348.

Aida, J., Y. Ando, R. Akhter, H. Aoyama, M. Masui und M. Morita (2006). „Reasons for permanent tooth extractions in Japan“, Journal of Epidemiology 16(5), 214-219.

Akifusa, S., I. Soh, T. Ansai, T. HAmasaki, Y. TAKata, A. Yohida et al. (2005). Relationship of number of remaining teeth to health-related quality of life in community-dwelling elderly. Gerodontology 22(2), 91-97.

Albandar, J. M., L. J. BROWN und H. LOE (1997). „Clinical features of early-onset periodontitis“, Journal of the American Dental Association 128(10), 1393-1399.

Al-Shammari, K. F., J. M. Al-Ansari, M. A. Al-Melh und A. K. Al-Khabbaz (2006). „Reasons for tooth extraction in Kuwait", Medical Principals and Practice 15(6), 417-422.

Alt, K. W., N. Nicklisch, P. Held, C. Meyer, A. Rossbach und M. Burwinkel. (2006). Zähne als Gesundheits- und Mortalitätsrisiko. Rahden.

Angelillo, I. F., C. G. Nobile und M. Pavia,(1996). „Survey of reasons for extraction of permanent teeth in Italy“, Community Dentistry and Oral Epidemiology 24(5), 336-340.

AZARPAZHOOH, A. und J. L. LEAKE (2006). „Systematic review of the association between respiratory diseases and oral health“, Journal of Periodontology 77(9), 1465-1482.

Baelum, V., W. van Palenstein Helderman, A. Hugoson, R. Yee und O. Fejerskov (2007). „A global perspective on changes in the burden of caries and periodontitis: implications for dentistry“, Journal of Oral Rehabilitation 34(12), 872-906; discussion 940.

Baratti-Mayer, D., B. Pittet, D. Montandon, I. Bolivar, J. E. Bornand, S. Hugonnet et al. (2003). „Noma: an „infectious“ disease of unknown aetiology“, The Lancet Infectious Disease 3(7), 419-431.

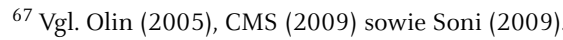


Bartzokas, C. A., R. Johnson, M. Jane, M. V. Martin, P. K. Pearce und Y. Saw (1994). "Relation between mouth and haematogenous infection in total joint replacements", British Medical Journal 309(6953), 506-508.

Beck, J., R. Garcia, G. Heiss, P. S. Vokonas und S. Offenbacher (1996). „Periodontal disease and cardiovascular disease“, Journal of Periodontology 67 (Supplement 10), 1123-1137.

Beikler, T., U. Peters, S. Prajaneh, K. Prior, B. Ehmke und T. F. Flemmig (2003). „Prevalence of Porphyromonas gingivalis fimA genotypes in Caucasians“, European Journal of Oral Sciences 111(5), 390-394.

Beikler, T., G. Abdeen, S. Schnitzer, S. Salzer, B. Ehmke, A. Heinecke et al. (2004a). „Microbiological shifts in intra- and extraoral habitats following mechanical periodontal therapy“, Journal of Clinical Periodontology 31(9), 777-783.

Beikler, T., K. Prior, B. EhmKe und T. F. Flemmig (2004b). „Specific antibiotics in the treatment of periodontitis - a proposed strategy“, Journal of Periodontology 75(1), 169-175.

Beikler, T., U. Peters, K. Prior, M. Eisenacher und T. F. Flemmig (2008). „Gene expression in periodontal tissues following treatment", BMC Medical Genomics 1, 30.

Bergstrom, J. (2004). „Tobacco smoking and chronic destructive periodontal disease“, Odontology $92(1), 1-8$.

Bomeli, S. R., B. F. BRAnstetter und B. J. Ferguson (2009). „Frequency of a dental source for acute maxillary sinusitis“, Laryngoscope 119(3), 580-584.

BRATTHALL, D. (2005). „Estimation of global DMFT for 12-year-olds in 2004“, International Dental Journal 55(6), 370-372.

CAhen, P. M., R. M. Frank und J. C. TuRlot (1985). „A survey of the reasons for dental extractions in France“, Journal of Dental Research 64(8), 1087-1093.

Chapple, I. L. (2009). „The impact of oral disease upon systemic health-Symposium overview“, Journal of Dentistry 37(8), S568-571.

Chauncey, H. H., R. L. Glass und J. E. Alman (1989). „Dental caries. Principal cause of tooth extraction in a sample of US male adults“, Caries Research, 23(3), 200-205.

Chen, T., Y. Hosogi, K. Nishikawa, K. Abbey, R. D. Fleischmann, J. Walling et al. (2004). "Comparative whole-genome analysis of virulent and avirulent strains of Porphyromonas gingivalis", Journal of Bacteriology 186(16), 5473-5479.

CMS (2009). National Health Expenditure Data: National Health Expenditures by Type of Service and Source of Funds, http://www.cms.gov/NationalHealthExpendData/ (18.11.2010).

Collin, H. L., M. Uusitupa, L. Niskanen, V. Kontturi-Narhi, H. MarkKanen, A. M. KoiviSTO et al. (1998). „Periodontal findings in elderly patients with non-insulin dependent diabetes mellitus“, Journal of Periodontology 69(9), 962-966.

Colombo, A. P., S. K. Boches, S. L. Cotton, J. M. Goodson, R. Kent, A. D. Haffajee et al. (2009). „Comparisons of subgingival microbial profiles of refractory periodontitis, severe periodontitis, and periodontal health using the human oral microbe identification microarray", Journal of Periodontology 80(9), 1421-1432.

Craig, R. G., J. K. Yip, M. K. So, R. J. Boylan, S. S. Socransky und A. D. Haffajee (2003). "Relationship of destructive periodontal disease to the acute-phase response“, Journal of Periodontology 74(7), 1007-1016.

DAvies, D. (2003). „Understanding biofilm resistance to antibacterial agents“, Nature Reviews. Drug Discovery 2(2), 114-122.

Deliargyris, E. N., P. N. Madianos, W. Kadoma, I. Marron, S. C. Smith Jr., J. D. Beck et al. (2004). „Periodontal disease in patients with acute myocardial infarction: prevalence and contribution to elevated C-reactive protein levels“, American Heart Journal 147(6), 1005-1009. 
Dewitte, S. N. und J. BeKVAlac (2009). "Oral health and frailty in the medieval English cemetery of St Mary Graces", American Journal of Physical Anthropology 142(3), 341-354.

Dietrich, T., M. Jimenez, E. A. Krall Kaye, P. S. Vokonas und R. I. Garcia (2008). „Agedependent associations between chronic periodontitis/edentulism and risk of coronary heart disease“, Circulation 117(13), 1668-1674.

Domejean-Orliaguet, S., S. A. Gansky und J. D. Featherstone (2006). „Caries risk assessment in an educational environment“, Journal of Dental Education 70(12), 1346-1354.

Dorfer, C. E., H. Becher, C. M. Ziegler, C. KAiser, R. Lutz, D. Jorss et al. (2004). „The association of gingivitis and periodontitis with ischemic stroke“, Journal of Clinical Periodontology 31(5), 396-401.

Dye, B. A., S. Tan, V. Smith, B. G. Lewis, L. K. Barker, G. Thornton-Evans et al. (2007). "Trends in oral health status: United States, 1988-1994 and 1999-2004“, Vital and Health Statistics 11(248), 1-92.

Elias, A. C. und A. Sheiham (1999). „The relationship between satisfaction with mouth and number, position and condition of teeth: studies in Brazilian adults", Journal of Oral Rehabilitation 26(1), 53-71.

Emrich, L. J., M. Shlossman und R. J. Genco (1991). „Periodontal disease in non-insulindependent diabetes mellitus“, Journal of Periodontology 62(2), 123-131.

EnWonwu, C. O., W. A. FAlKLeR und E. O. Idigbe (2000). „Oro-facial gangrene (noma/cancrum oris): pathogenetic mechanisms", Critical Reviews in Oral Biology and Medicine 11(2), 159171.

Ezzo, P. J. und C. W. CuTLER (2003). „Microorganisms as risk indicators for periodontal disease“, Periodontology 2000 32, 24-35.

Faveri, M., L. C. Figueiredo, P. M. Duarte, M. J. Mestnik, M. P. Mayer und M. Feres (2009). „Microbiological profile of untreated subjects with localized aggressive periodontitis“, Journal of Clinical Periodontology 36(9), 739-749.

FEnG, Z. und A. Weinberg (2006). „Role of bacteria in health and disease of periodontal tissues“, Periodontology 2000 40, 50-76.

Fitzpatrick, S. G. und J. Katz (2009). „The Association Between Periodontal Disease and Cancer: A Review of the Literature“, Journal of Dentistry 38(2), 83-95.

Flemmig, T. F. (1999). „Periodontitis“, Annals of Periodontology 4(1), 32-38.

ForshaW, R. J. (2009). „Dental health and disease in ancient Egypt“, British Dental Journal 206(8), 421-424.

Fraser, C., W. P. HANAGE und B. G. SPRATt (2005). „Neutral microepidemic evolution of bacterial pathogens", Proceedings of the National Academy of Sciences of the United States of America 102(6), 1968-1973.

GARCIA, R. (2009). „Periodontal treatment could improve glycaemic control in diabetic patients“, Evidence-based Dentistry 10(1), 20-21.

Gotfredsen, K. und A. W. Walls (2007). „What dentition assures oral function?“, Clinical Oral Implants Research 18 (Supplement 3), 34-45.

Grossi, S. G. (1994). „Assessment of risk for periodontal disease. I. Risk indicators for attachment loss“, Journal of Periodontology 65, 260.

Grossner-Schreiber, B., J. Teichmann, M. Hannig, C. Dorfer, D. F. Wenderoth und S. J. ОTT (2009). „Modified implant surfaces show different biofilm compositions under in vivo conditions“, Clinical Oral Implants Research 20(8), 817-826.

Gutmann, J. L., J. C. Baumgartner, A. H. Gluskin, G. R. Hartwell und R. E. Walton (2009). „Identify and define all diagnostic terms for periapical/periradicular health and disease States", Journal of Endodontics 35(12), 1658-1674. 
Haffajee, A. D., S. S. Socransky, C. Smith und S. Dibart (1991). „Relation of baseline microbial parameters to future periodontal attachment loss", Journal of Clinical Periodontology 18(10), 744-750.

Haffajee, A. D., Socransky, S. S., M. R. Patel und X. Song (2008). „Microbial complexes in supragingival plaque“, Oral Microbiology and Immunology 23(3), 196-205.

Haubek, D., O. K. Ennibi, K. Poulsen, N. Benzarti und V. Baelum (2004). „The highly leukotoxic JP2 clone of Actinobacillus actinomycetemcomitans and progression of periodontal attachment loss“, Journal of Dental Research 83(10), 767-770.

Haubek, D., K. Poulsen und M. Kilian (2007). „Microevolution and patterns of dissemination of the JP2 clone of Aggregatibacter (Actinobacillus) actinomycetemcomitans", Infection and Immunity 75(6), 3080-3088.

HeitZ-MAYField, L. J. (2005). „Disease progression: identification of high-risk groups and individuals for periodontitis“, Journal of Clinical Periodontology 32 (Supplement 6), 196-209.

Hintao, J., R. Teanpaisan, V. Chongsuvivatwong, C. Ratarasan und G. Dahlen (2007). „The microbiological profiles of saliva, supragingival and subgingival plaque and dental caries in adults with and without type 2 diabetes mellitus", Oral Microbiology and Immunology 22(3), 175-181.

Hojo, K., S. Nagaoka, T. Ohshima und N. Maeda (2009). „Bacterial interactions in dental biofilm development", Journal of Dental Research 88(11), 982-990.

Holt, S. C. und J. L. Ebersole (2005). Porphyromonas gingivalis, Treponema denticola, and Tannerella forsythia: the „red complex“, a prototype polybacterial pathogenic consortium in periodontitis. Periodontology 2000 38, 72-122.

Hotopp, J. C., R. Grifantini, N. Kumar, Y. L. Tzeng, D. Fouts, E. Frigimelica et al. (2006). "Comparative genomics of Neisseria meningitidis: core genome, islands of horizontal transfer and pathogen-specific genes", Microbiology 152(Pt 12), 3733-3749.

Hugoson, A., G. Koch, C. GothberG, A. N. Helkimo, S. A. Lundin, O. Norderyd et al. (2005a). "Oral health of individuals aged 3-80 years in Jonkoping, Sweden during 30 years (19732003). I. Review of findings on dental care habits and knowledge of oral health", Swedish Dental Journal 29(4), 125-138.

Hugoson, A., G. Koch, C. Gothberg, A. N. Helkimo, S. A. Lundin, O. Norderyd et al. (2005b). "Oral health of individuals aged 3-80 years in Jonkoping, Sweden during 30 years (19732003). II. Review of clinical and radiographic findings“, Swedish Dental Journal 29(4), 139 155.

Imazato, S., K. Ikebe, T. Nokubi, S. Ebisu und A. W. Walls (2006). „Prevalence of root caries in a selected population of older adults in Japan“, Journal of Oral Rehabilitation 33(2), 137-143.

Inaba, H., K. NaKano, T. Kato, R. Nomura, S. KaWAi, M. Kuboniwa et al. (2008). „Heterogenic virulence and related factors among clinical isolates of Porphyromonas gingivalis with type II fimbriae“, Oral Microbiology and Immunology 23(1), 29-35.

KAY, E. J. und A. S. BLINKHORN (1986). „The reasons underlying the extraction of teeth in Scotland“, British Dental Journal 160(8), 287-290.

Khader, Y. S., A. S. Dauod, S. S. El-Qaderi, A. Alkafajei und W. Q. Batayha (2006). „Periodontal status of diabetics compared with nondiabetics: a meta-analysis“, Journal of Diabetes and its Complications 20(1), 59-68.

KIM, J. und S. AMAR (2006). „Periodontal disease and systemic conditions: a bidirectional relationship“, Odontology 94(1), 10-21.

KLOCK, K. S. und O. Haugejorden (1991). „Primary reasons for extraction of permanent teeth in Norway: changes from 1968 to 1988“, Community Dentistry and Oral Epidemiology 19(6), 336-341. 
Kolenbrander, P. E., R. N. Andersen, D. S. Blehert, P. G. Egland, J. S. Foster und R. J. PalMER Jr. (2002). „Communication among oral bacteria“, Microbiology and Molecular Biology Reviews: MMBR 66(3), 486-505, table of contents.

Langsjoen, O. (Hrsg., 1998). Diseases of the Dentition. Cambridge.

LARSEN, C. S. (1995). „Biological changes in human populations with agriculture“, Annual Review of Anthropology 24, 185-213.

Le Pera, A. F., R. A. Mahevich und H. Silverstein (2005). „Xerostomia and its effects on the dentition", Journal of the New Jersey Dental Association 76(2), 19-21.

LeEK, F. F. (1966). „Observations on the Dental Pathology Seen in Ancient Egyptian Skulls“, The Journal of Egyptian Archaeology 52, 59-64.

Levin, L. G., A. S. Law, G. R. Holland, P. V. AввотT und R. S. Roda (2009). „Identify and define all diagnostic terms for pulpal health and disease States“, Journal of Endodontics 35(12), 16451657.

Li, X., K. M. Kolltveit, L. Tronstad und I. Olsen (2000). „Systemic diseases caused by oral infection“, Clinical Microbiology Reviews 13(4), 547-558.

Lim, L. P., F. B. TAY, C. F. Sum und A. C. ThAi (2007). „Relationship between markers of metabolic control and inflammation on severity of periodontal disease in patients with diabetes mellitus", Journal of Clinical Periodontology 34(2), 118-123.

Linden, G. J., K. McClean, I. YounG, A. Evans und F. Kee (2008). „Persistently raised C-reactive protein levels are associated with advanced periodontal disease", Journal of Clinical Periodontology 35(9), 741-747.

Loos, B. G., R. P. JoHN und M. L. LAINE (2005). „Identification of genetic risk factors for periodontitis and possible mechanisms of action“, Journal of Clinical Periodontology 32, 159.

Machtei, E. E., R. Dunford, E. Hausmann, S. G. Grossi, J. Powell, D. Cummins et al. (1997). "Longitudinal study of prognostic factors in established periodontitis patients“, Journal of Clinical Periodontology 24(2), 102-109.

Machtei, E. E., E. Hausmann, R. Dunford, S. Grossi, A. Ho, G. Davis et al. (1999). „Longitudinal study of predictive factors for periodontal disease and tooth loss“, Journal of Clinical Periodontology 26(6), 374-380.

MARCK, K. W. (2003). „A history of noma, the "Face of Poverty" ${ }^{\text {'“ }}$ Plastic and Reconstructive Surgery 111(5), 1702-1707.

Mariotti, A. (1999). Dental plaque-induced gingival diseases. Annals of Periodontology 4(1), 719.

MCCAUL, L. K., W. M. JENKINS und E. J. KAY (2001). „The reasons for extraction of permanent teeth in Scotland: a 15-year follow-up study", British Dental Journal 190(12), 658-662.

MeAley, B. (2000). „American Academy of Periodontology Position Paper - Diabetes and periodontal diseases“, Journal of Periodontology 71, 664.

Mealey, B. L. und T. W. OAtes (2006). „Diabetes mellitus and periodontal diseases“, Journal of Periodontology 77(8), 1289-1303.

MenG, H. X. (1999a). „Periodontal abscess“, Annals of Periodontology 4(1), 79-83.

MenG, H. X. (1999b). „Periodontic-endodontic lesions“, Annals of Periodontology 4(1), 84-90.

Michalowicz, B. S., S. R. Diehl, J. C. Gunsolley, B. S. Sparks, C. N. Brooks, T. E. Koertge et al. (2000). „Evidence of a substantial genetic basis for risk of adult periodontitis“, Journal of Periodontology 71(11), 1699-1707.

Mignogna, M. D. und S. Fedele (2006). „The neglected global burden of chronic oral diseases“, Journal of Dental Research 85(5), 390-391. 
Mombelli, A., F. CASAgni und P. N. Madianos (2002). „Can presence or absence of periodontal pathogens distinguish between subjects with chronic and aggressive periodontitis? A systematic review“, Journal of Clinical Periodontology 29 (Supplement 3), 10-21 und 37-18.

Mueller, A. A., B. Saldamli, S. Stubinger, C. Walter, U. Fluckiger, A. Merlo et al. (2009). "Oral bacterial cultures in nontraumatic brain abscesses: results of a first-line study", Oral Surgery, Oral Medicine, Oral Pathology, Oral Radiology and Endodontics 107(4), 469-476.

MurRay, H., M. ClARKe, D. LOCKER und E. J. KAy (1997). „Reasons for tooth extractions in dental practices in Ontario, Canada according to tooth type", International Dental Journal 47(1), 3-8.

Mylonas, A. I., F. H. TZerbos, M. Mihalaki, D. Rologis und I. BoutsiKakis (2007). „Cerebral abscess of odontogenic origin“, Journal of Cranio-maxillo-facial Surgery 35(1), 63-67.

Nelson, R. G., M. Shlossman, L. M. Budding, D. J. Pettitt, M. F. SaAd, R. J. Genco et al. (1990). „Periodontal disease and NIDDM in Pima Indians“, Diabetes Care 13(8), 836-840.

Nishinara, T. und T. Koseki (2004). „Microbial etiology of periodontitis“, Periodontology 2000 $36,14-26$.

NovaK, M. J. (1999). „Necrotizing ulcerative periodontitis“, Annals of Periodontology 4(1), 74-78.

OLin Gary L. und Jeffrey A. RHOAdes (2005). „The five most costly medical conditions, 1997 and 2002: Estimates for the U.S. civilian noninstitutionalized population“, Medical Expenditure Panel Survey, Statistical Brief \#80.

ONG, G., J. F. YeO und S. Bhole (1996). „A survey of reasons for extraction of permanent teeth in Singapore“, Community Dentistry and Oral Epidemiology 24(2), 124-127.

PAlmer, R. J. (2009). „Oral bacterial biofilms - history in progress“, Microbiology 155(Pt 7), 2113 2114.

Parahitiyawa, N. B., L. J. Jin, W. K. Leung, W. C. Yam und L. P. SAmaranayake (2009). „Microbiology of odontogenic bacteremia: beyond endocarditis“, Clinical Microbiology Reviews 22(1), 46-64, Table of Contents.

Persson, G. R. und R. E. Persson (2008). „Cardiovascular disease and periodontitis: an update on the associations and risk“, Journal of Clinical Periodontology 35 (Supplement 8), 362-379.

Petersen, P. E., D. Bourgeois, H. Ogawa, S. Estupinan-Day und C. Ndiaye (2005). „The global burden of oral diseases and risks to oral health", Bulletin of the World Health Organization 83(9), 661-669.

Phipps, K. R. und V. J. Stevens (1995). „Relative contribution of caries and periodontal disease in adult tooth loss for an HMO dental population“, Journal of Public Health Dentistry 55(4), $250-252$.

Ramage, G., E. Mowat, B. Jones, C. Williams und J. Lopez-Ribot (2009). „Our current understanding of fungal biofilms", Critical Reviews in Microbiology 35(4), 340-355.

Reich, E. und K. A. Hiller (1993). „Reasons for tooth extraction in the western states of Germany“, Community Dentistry and Oral Epidemiology 21(6), 379-383.

Richards, W., J. Ameen, A. M. Coll und G. Higgs (2005). „Reasons for tooth extraction in four general dental practices in South Wales“, British Dental Journal 198(5), 275-278.

Riviere, G. R., K. S. Smith, E. Tzagaroulaki, S. L. Kay, X. Zhu, T. A. DeRouen et al. (1996). „Periodontal status and detection frequency of bacteria at sites of periodontal health and gingivitis“, Journal of Periodontology 67(2), 109-115.

RobERTSON, D. und A. J. SMith (2009). „The microbiology of the acute dental abscess“, Journal of Medical Microbiology 58(Pt 2), 155-162.

Rocha, C. T., M. A. Rossi, M. R. Leonardo, L. B. Rocha, P. Nelson-Filho und L. A. Silva (2008). „Biofilm on the apical region of roots in primary teeth with vital and necrotic pulps with or without radiographically evident apical pathosis", International Endodontic Journal 41(8), 664-669. 
Sachdeo, A., A. D. Haffajee und S. S. Socransky (2008). „Biofilms in the edentulous oral cavity", Journal of Prosthodontics 17(5), 348-356.

Salvi, G. E., B. Carollo-Bittel und N. P. LANG (2008). „Effects of diabetes mellitus on periodontal and peri-implant conditions: update on associations and risks“, Journal of Clinical Periodontology 35 (Supplement 8), 398-409.

SAndoval-Montes, C. und L. SAntos-ARgumedo (2005). „CD38 is expressed selectively during the activation of a subset of mature $\mathrm{T}$ cells with reduced proliferation but improved potential to produce cytokines". Journal of Leukocyte Biology 77, 513-521.

Schaudinn, C., A. Gorur, D. Keller, P. P. Sedghizadeh und J. W. Costerton (2009). „Periodontitis: an archetypical biofilm disease", Journal of the American Dental Association 140(8), 978-986.

SHARKAWY, A. A. (2007). „Cervicofacial actinomycosis and mandibular osteomyelitis“, Infectious Disease Clinics of North America 21(2), 543-556, viii.

Sheiham, A., J. G. Steele, W. Marcenes, G. Tsakos, S. Finch und A. W. Walls (2001). „Prevalence of impacts of dental and oral disorders and their effects on eating among older people; a national survey in Great Britain“, Community Dentistry and Oral Epidemiology 29(3), 195-203.

Silva, N., N. Dutzan, M. Hernandez, A. Dezerega, O. Rivera, J. C. Aguillon et al. (2008). "Characterization of progressive periodontal lesions in chronic periodontitis patients: levels of chemokines, cytokines, matrix metalloproteinase-13, periodontal pathogens and inflammatory cells", Journal of Clinical Periodontology 35(3), 206-214.

Slade, G. D., S. Offenbacher, J. D. Beck, G. Heiss und J. S. Pankow (2000). „Acute-phase inflammatory response to periodontal disease in the US population“, Journal of Dental Research 79(1), 49-57.

SLOTS, J. und M. TING (1999). „Actinobacillus actinomycetemcomitans and Porphyromonas gingivalis in human periodontal disease: occurrence and treatment", Periodontology 200020 , $82-121$.

Socransky, S. S. und A. D. HAfFajee (1992). „The bacterial etiology of destructive periodontal disease: current concepts“, Journal of Periodontology 63 (Supplement 4), 322-331.

Socransky, S. S., A. D. Haffajee, M. A. Cugini, C. Smith und R. L. Kent Jr. (1998). „Microbial complexes in subgingival plaque", Journal of Clinical Periodontology 25(2), 134-144.

Socransky, S. S. und A. D. Haffajee (2005). „Periodontal microbial ecology“, Periodontology $200038,135-187$.

Soni, Anita (2009). „The five most costly conditions, 1996 and 2006: Estimates for the U.S. civilian noninstitutionalized population", Medical Expenditure Panel Survey, Statistical Brief \#248.

Stephens, R. G., S. L. Kogon und A. M. JARVis (1991). „A study of the reasons for tooth extraction in a Canadian population sample“, Journal (Canadian Dental Association) 57(6), 501-504.

TABOR, H. K., N. J. RisCH und R. M. MYERS (2002). „Candidate-gene approaches for studying complex genetic traits: practical considerations“, Nature Reviews: Genetics 3(5), 391-397.

TAN, K. S., K. P. SONG und G. ONG (2002). „Cytolethal distending toxin of Actinobacillus actinomycetemcomitans. Occurrence and association with periodontal disease“, Journal of Periodontal Research 37(4), 268-272.

Tanner, A. C., R. Kent Jr., E. Kanasi, S. C. Lu, B. J. Paster, S. T. Sonis et al. (2007). „Clinical characteristics and microbiota of progressing slight chronic periodontitis in adults", Journal of Clinical Periodontology 34(11), 917-930.

TAYLOR, G. W. (1998). „Glycaemic control and alveolar bone loss progression in type 2 diabetes“, Annals of Periodontology 3, 30. 
Teles, R. P., M. Patel, S. S. Socransky und A. D. Haffajee (2008). „Disease progression in periodontally healthy and maintenance subjects“, Journal of Periodontology 79(5), 784-794.

TEMPLE, D. H. und C. S. LARSEN (2007). „Dental caries prevalence as evidence for agriculture and subsistence variation during the Yayoi period in prehistoric Japan: biocultural interpretations of an economy in transition", American Journal of Physical Anthropology 134(4), 501-512.

Tettelin, H., V. Masignani, M. J. Cieslewicz, C. Donati, D. Medini, N. L. Ward et al. (2005). „Genome analysis of multiple pathogenic isolates of Streptococcus agalactiae: implications for the microbial 'pan-genome'", Proceedings of the National Academy of Sciences of the United States of America 102(39), 13950-13955.

Timmerman, M. F., G. A. Van der Weijden, F. Abbas, E. M. Arief, S. Armand, E. G. Winkel et al. (2000). „Untreated periodontal disease in Indonesian adolescents. Longitudinal clinical data and prospective clinical and microbiological risk assessment", Journal of Clinical Periodontology 27(12), 932-942.

Tonetti, M. S. (2009). „Periodontitis and risk for atherosclerosis: an update on intervention trials“, Journal of Clinical Periodontology 36 (Supplement 10), 15-19.

Tonetti, M. S. und A. Mombelli (1999). „Early-onset periodontitis“, Annals of Periodontology $4(1), 39-53$.

Toomes, C., J. James, A. J. Wood, C. L. Wu, D. McCormick, N. LenCH et al. (1999). „Loss-offunction mutations in the cathepsin $\mathrm{C}$ gene result in periodontal disease and palmoplantar keratosis“, Nature Genetics 23(4), 421-424.

Topazian, Richard G., Morton H. Goldberg und James R. Hupp ( $\left.{ }^{4} 2002\right)$. Oral and Maxillofacial Infections. Philadelphia.

Tran, S. D., J. D. Rudney, B. S. Sparks und J. S. Hodges (2001). „Persistent presence of Bacteroides forsythus as a risk factor for attachment loss in a population with low prevalence and severity of adult periodontitis“, Journal of Periodontology 72(1), 1-10.

TroviK, T. A., K. S. KLOCK und O. HAugejorden (2000). „Trends in reasons for tooth extractions in Norway from 1968 to $1998^{\prime \prime}$, Acta odontologica Scandinavica 58(2), 89-96.

TSAi, C., C. HAyES und G. W. TAYLOR (2002). „Glycemic control of type 2 diabetes and severe periodontal disease in the US adult population“, Community Dentistry and Oral Epidemiology 30(3), 182-192.

Ulivieri, S., G. Oliveri und G. Filosomi (2007). „Brain abscess following dental procedures. Case report“, Minerva Stomatologica 56(5), 303-305.

Vieira, F., S. M. Allen, R. M. Stocks und J. W. Thompson (2008). „Deep neck infection“, Otolaryngol Clinics of North America 41(3), 459-483, vii.

Wagner, K. W., R. Schon, M. Schumacher, R. Schmelzeisen und D. Schulze (2006). „Case report: brain and liver abscesses caused by oral infection with Streptococcus intermedius", Oral Surgery, Oral Medicine, Oral Pathology, Oral Radiology and Endodontics 102(4), e21-23.

WHO. (2009a). http://www.dent.niigata-u.ac.jp/prevent/perio/contents.html (10.09.2010).

WHO. (2009b). http://www.whocollab.od.mah.se/expl/globalcar1.html (01.04.2010).

Wilson, W., K. A. Taubert, M. Gewitz, P. B. Lockhart, L. M. Baddour, M. Levison et al. (2007). „Prevention of infective endocarditis: guidelines from the American Heart Association: a guideline from the American Heart Association Rheumatic Fever, Endocarditis, and Kawasaki Disease Committee, Council on Cardiovascular Disease in the Young, and the Council on Clinical Cardiology, Council on Cardiovascular Surgery and Anesthesia, and the Quality of Care and Outcomes Research Interdisciplinary Working Group“, Circulation 116(15), 1736-1754.

Wimmer, G. und B. L. Pihlstrom (2008). „A critical assessment of adverse pregnancy outcome and periodontal disease“, Journal of Clinical Periodontology 35 (Supplement 8), 380-397. 
Ximenez-Fyvie, L. A., A. D. Haffajee und S. S. Socransky (2000). „Comparison of the microbiota of supra- and subgingival plaque in health and periodontitis", Journal of Clinical Periodontology 27(9), 648-657.

Yoshida, Y., Y. Hatanaka, M. Imaki, Y. Ogawa, S. MiYatani und S. TANAda (2001). „Epidemiological study on improving the QOL and oral conditions of the aged - Part 1: The relationship between the status of tooth preservation and QOL", Journal of Physiological Anthropology and Applied Human Science 20(6), 363-368.

ZitZMANn, N. U. und T. BERGLUndh (2008). „Definition and prevalence of peri-implant diseases“, Journal of Clinical Periodontology 35 (Supplement 8), 286-291. 



\section{Prof. Dr. Matthias Schott}

Matthias Schott studierte in den Jahren 1989 bis 1996 Humanmedizin an den Universitäten Leipzig, Cambridge und Bordeaux. Im Jahre 1993 erhielt er ein einjähriges DAAD-Stipendium für einen Forschungsaufenthalt in den USA. Seine Facharztausbildung begann er im Jahre 1996 und beendet sie 2003 in Düsseldorf. Im Jahre 2003 folgte die Habilitation sowie anschließend die Teilgebietsbezeichnungen Endokrinologie und Diabetologie. Im Jahre 2008 wurde er zum außerplanmäßigen Professor und im Jahre 2010 zum Universitätsprofessor (W2) für Endokrinologie ernannt.

Sein Forschungsinteresse liegt auf den Gebieten der Antitumorimmunität und der Autoimmunität. Er veröffentlichte eine Vielzahl an Publikationen in hochrangigen Wissenschaftsjournalen wie zum Beispiel The Lancet. Er ist Mitglied in vielen wissenschaftlichen Organisationen, einschließlich einer Mitgliedschaft im Wissenschaftlichen Beirat der Amerikanischen Schilddrüsengesellschaft. Im Jahre 2007 erhielt er den europäischen Schilddrüsenforschungspreis. Seit 2008 ist er Herausgeber des renomierten Year Book of Endocrinology. 